\title{
Das BKA im Wandel: Einst Zentralstelle, bald Bundeskrimi- nalpolizei im Vorfeld terroristischer Gefahren - die Große Koalition leitet einen Paradigmenwechsel ein
}

Im Juli 2007 legt die Bundesregierung einen ersten - bis heute unveröffentlichten - Referentenentwurf des „Gesetzes zur Abwehr von Gefahren des internationalen Terrorismus durch das Bundeskriminalamt" (nachfolgend Reformgesetz sowie Gesetzentwurf genannt und BKA - GE abgekürzt) vor. Es soll das „Gesetz über die Zusammenarbeit des Bundes und der Länder in kriminalpolizeilichen Angelegenheiten " (Bundeskriminalamtgesetz - im folgenden BKAG genannt) reformieren. Nach starker Kritik von Medien und Bürgerrechtsorganisationen ${ }^{1}$ verabschiedet das Bundeskabinett am 04. Juni 2008 dessen zwischenzeitlich mit den Innenministerien der Länder erneut abgestimmte Fassung. ${ }^{2}$ Sechzehn Tage später berät der Bundestag diese in erster Lesung als Gesetzesentwurf. ${ }^{3}$ Am 15 . September hört sein Innenausschuss Sachverständige zum Entwurf an. ${ }^{4}$ Es stellt sich zunächst die Frage nach dem späten Zeitpunkt dieser Novelle: Sie erfolgt im 7. Jahr nach dem 11. September. Betont nicht aber das Bundesinnenministerium (BMI) seitdem eine fortwährend gegebene abstrakte Terrorgefahr?

Angeblich wäre die Gefahr von Anschlägen permanent groß: Doch erst sieben Jahre nach dem 11. September kommt es zum Umbau des BKA - Welche Gründe hat dies?

Wieso kommt es erst sieben Jahre nach den Anschlägen vom 11. September 2001 zu einer derartig umfassenden Novelle? Der Grund läge laut BMI in einem einzigen Ermittlungsverfahren verortet: Betreffend der letztes Jahr aufgedeckten islamistischen „Sauerländer Zelle" habe die Bundesregierung Erkenntnisse über Fehlabläufe und Mängel bei verdeckten präventiven Überwachungsmaßnahmen gewonnen. Deren schwerwiegender Charakter rechtfertige die Implementierung entsprechender Befugnisse. ${ }^{5}$ Und so umfasst der Katalog von für das BKAG vorgesehener neuer Eingriffsvorschriften u.a. die Rasterfahndung, die Überwachung der Telekommunikation, Lausch- und Spähangriffe, den Einsatz von IMSI - Catchern, abgesenkte Tatbestände für Datenaustausch mit in- und ausländischen Nachrichtendiensten, umstrittene verdeckte Eingriffe in informationstechnische Systeme, Platzverweise zur Absicherung von Observationen sowie großzügige Ausnahmetatbestände, die das Absehen der Benachrichtigung der von heimlichen Maßnahmen Betroffenen gestatten. ${ }^{6}$ Derartige Befugnisse sollen demnach der Förderalismusreform gemäß Art 73 Nr. 9a GG für die Abwehr länderübergreifender terroristischer Gefahren zuständigen Wiesbadener Kriminalamt straflose Vorbereitungshandlungen von nicht ins Versuchsstadium eingetretenen Organisations-, Sprengstoff- bzw. Gewaltdelikten erschließen: Man hofft, terroristische Vereinigungen frühzeitig zu erkennen. Fraglich erscheint, ob es sich bei dieser eindeutig strafverfahrensinitiierend auf die Suche nach Verdachtsmomenten i.S.d.

\129a StGB angelegten pro- aktiven Kriminalstrategie noch um Gefahrenabwehr handelt. Innenpolitiker der Regierung behaupten, die Novelle verleihe dem BKA lediglich Befugnisse, über welche die
Länderpolizeien seit langem verfügten, so dass es nun auch Gefahren verhüten könne. ${ }^{7}$ Doch die im BKA - GE enthaltenen Befugnisse über heimliche Ermittlungen weisen tatbestandliche Schwellen auf, welche das Vorfeld der konkreten Gefahr erschließen. Das wirft die Frage auf, ob die Bundesregierung ihr Kriminalamt in eine Art von geheim Erkenntnisse ermittelnden Dienst umgestaltet: Ähnelt dessen Charakter zukünftig dem eines über Exekutivbefugnisse verfügenden Nachrichtendienstes? Wenn ja, welche Kontroll- und Rechtsschutzmöglichkeiten sieht man gegen Maßnahmen dieser neuen Bundeskripo vor? Zur Untersuchung dieser Problemstellungen analysiert der Verfasser ${ }^{8}$ die grundlegende Systematik des im Bundestag am 20. Juni erstmals beratenen Reformgesetzes. Daneben bezieht er Stellungnahmen von Sachverständigen in seine Überlegungen ein.

Fundament des neuen Amtes: Mit welcher rechtstechnischen Konstruktion erschließt man das Vorfeld konkreter terroristischer Gefahren?

Geschickt verknüpfen die Autoren des Gesetzes zwei materielle Elemente mit einem flexiblen formellen Element, dem in $\$ 20$ BKA - GE enthaltenen Katalog von Befugnisnormen. Als eingriffsrechtlicher Joker bildet er den Motor der zukünftig für das Amt vorgesehenen pro - aktiven Funktion, Verfahren nach $\$ 129$ a StGB zu initiieren.

a) Erstes materielles Element: Der bisher verwendete Gefährderbegriff erfährt eine Expansion

\20b Abs. 2 Nr. 1 des BKA - GE enthält das erste von drei Elementen, eine Gefährderdefinition. Sie erfährt als abgewandelte (nämlich auf $\$ 129$ a StGB und nicht als Ursprungsdefinition des BMI auf den Katalog des $\$ 100$ a StPO gestützte und somit tatbestandlich engere) Definition der fragwürdigen Konstruktion politisch motivierter „Gefährder“ Verwendung. Was bedeutet seine Abwandlung?

aa) Erweiterter Gefährderbegriff des $\ 4 a:$ „Gefährder“ i.S.d. $\$ 129 a$ StGB!

Der bereits ein halbes Jahr nach dem 11. September 2001 von den Landeskriminalämtern zur materiellen Rechtfertigung von sog. „Gefahrenermittlungen“ ihrer Staatsschutzabteilungen verwendete Begriff ist bisher gesetzlich nicht geregelt. Erst im Jahre 2004 von der AG Kripo, einem Gremium des BMI, festgelegt, expandiert er nun tatbestandlich: $\mathbb{\int} 4 \mathrm{a}$ Abs. 1 Satz 2 BKA - GE weist dem BKA das Ermessen zu, „Straftaten zu verhüten, die in $\ 129$ a Abs. 1 und $2(. .$. )“ des Strafgesetzbuches bezeichnet sind und weiteren einschränkenden Kriterien unterliegen müssen. Diese Aufgabenzuweisung ist im Kontext mit $\ 20$ b Abs. 2 Nr. 1 BKA - GE zu lesen: „Zur Verhütung von Straftaten gemäß $\$ 4 a$ Abs. 1 Satz 2 ist eine Erhebung personenbezogener Daten nur zulässig, soweit Tatsachen die Annahme rechtfertigen, dass 1. die Person eine Straftat gemäß \4a Abs. 1 Satz 2 begehen will (...). “ $\$ 4$ a Abs. 1 Satz 2 BKA - GE wiederum verweist auf $\ 129$ a Abs. I u. II StGB, während die bisherige Gefährderdefinition den Katalog des $\ 100$ a StPO umfasst. Das macht einen Ver- 
gleich jener prozessualen Norm mit der materiellen Vorschrift des \129a StGB, die eine starke prozessuale Funktion hat (Teile der Lit. bezeichnet ihn als sog. „Türöffner“), nötig: Der Katalog des $\ 100 \mathrm{a}$ StPO ist zwar quantitativ weiter gefasst, enthält jedoch mehr Delikte. Doch hängen seine Maßnahmen zunächst von einer richterlichen Prüfung ab, während für ein Verfahren nach $\$ 129$ a StGB der Polizei schon ein Anfangsverdacht ausreicht, worauf Ermittlungen beginnen. Daneben umfasst $\ 129$ a StGB auch niedrigschwellige Delikte wie die Sachbeschädigung, während $\$ 100$ a StPO Delikte von mittelschwerer und schwerer Kriminalität aufzählt. Dieser Unterschied soll Gefahrenprognosen, wonach Taten i.S.d. $\int 4 a$ Abs. 1 S. 2 BKA - GE im Raume stünden, mehr Spielraum eröffnen, um Personen als „Gefährder des internationalen Terrorismus“ einzustufen und nach \20b Abs. 2 Nr. 1 i.V.m. \4a Abs. 1 S. 2 BKA - GE, also mit intensiven heimlichen Ermittlungen, deren personenbezogene Daten zu erheben. Daneben lässt sich als zweites materielles Fundament des Referentenentwurfes der Einfluss nachrichtendienstlicher Erkenntnisse auf kriminalpolizeiliche Gefahrenprognosen herauslesen, die eingestuften „Gefährdern“ deren Gefahrenqualität zuschreiben.

b) Zweites materielles Element: Kriminalitätsorientierte Gefahrenprognosen der Polizei

Die materielle Begründung polizeirechtlicher Eingriffe erfordert das möglichst auf eine Person bezogen prognostizierte Vorliegen eines länderübergreifenden Gefahrensachverhalts des internationalen Terrorismus. Sein Tatsachengehalt muss die Prognose begründen, wonach kraft des Verhaltens von Personen terroristische Schwerkriminalität Schutzgüter der Öffentlichen Sicherheit schädigen werde: Derartige Vorhersagen über mögliche Kriminalität begründen die materielle Rechtfertigungsfähigkeit von (heimlichen) präventiven Ermittlungen des BKA. Ohne sie kommt es nicht zur Rechtfertigung seiner Zuständigkeit. Sie sind von grundsätzlicher Bedeutung für kriminalpolizeiliche Vorfeldarbeit. Doch sie bilden weder gerichtsfeste Erkenntnisse, noch gesicherte polizeiliche Tatsachenlagen ab. Dem mit der Novelle über neue Befugnisse verfügenden BKA eröffnen diese jedoch heimliche Eingriffe gegenüber Menschen, die nicht Störer im Sinne des Polizeirechts sind. Und gerade jenes ist Ziel dieser Reform: Auf einem erweiterten Gefährderbegriff fußend, richtet das BMI die neuen Befugnisse seines Kriminalamtes auf das Vorfeld konkreter terroristischer Gefahren aus. Bedeutet diese pro - aktive Neuorientierung aber noch Gefahrenabwehr?

\section{Das formelle Element: Ermittlungsbefugnisse nach $\mathbb{} 20$ BKA - GE - Gefahrenabwehr?}

Die in $\ 20$ d.E. aufgeführten Maßnahmen dienen der Früherkennung islamistischer Zellen, von denen „Gefahren des internationalen Terrorismus“ ausgehen können. Personen, die zukünftig vielleicht terroristische Straftaten begehen könnten, sollen erkannt werden: Es handele sich um Befugnisse zur Verhütung von Straftaten, also um polizeiliche Gefahrenabwehr. Doch trifft das zu? Laut Gesetzgeber sollen $\mathbb{2 0} \mathrm{a}-\mathrm{x}$ BKA - GE dem BKA die Abwehr terroristischer Gefahren ermöglichen. ${ }^{9}$ Das überzeugt auf den ersten Blick: Zählt doch die Ermittlung länderübergreifender Gefahrensachverhalte des internationalen Terrorismus kraft der Novelle ${ }^{10}$ des Art. 73 Nr. 9a GG zur neuen Kompetenz des Amtes: Dem BKA obliegen in jenen eng begrenzten Fällen präventive Ermittlungen zur Abwehr von aus islamistischer Schwerkriminalität hervorgehenden Gefahren. In dem Zusammenhang sollen es die angedachten Befugnisse ermöglichen, für jene Gefahren verantwortliche Personen, deren Kontaktzusammenhänge sowie ggf. Tatplanungen aufzudecken. Hält man sich vor
Augen, dass terroristische Kriminalität oftmals hohe Opferzahlen fordert, klingt dieser Ansatz, wonach ein als möglich prognostiziertes zukünftiges Ereignis verhütet werden soll, schlüssig. Doch ein Umstand lässt an der Subsumtion dieser Maßnahmen unter die polizeiliche Aufgabe „Gefahrenabwehr“ zweifeln: Das Verhältnis der konkreten Gefahr des Polizeirechts zum strafprozessualen Anfangsverdacht. Es wird vor allem im Phänomenbereich aller Arten von Organisationskriminalität von einer Kongruenz geprägt: Liegt eine Gefahrenprognose vor, wonach ein Anschlag möglich ist, verkörpert diese Information einerseits einen Sachverhalt mit Tatsachenverdichtung, eine konkrete Gefahr. Andererseits entspricht jene Verdichtung den tatbestandlichen Anforderungen des strafprozessualen Anfangsverdachts: Denn bezüglich des prognostizierten Schadensereignisses sind zeitlich und bzw. oder örtlich bestimmte Anhaltspunkte gegeben, wonach a) eine Tat verwirklicht werde und b) eine terroristische Vereinigung bestehen könnte, aus der ihre Planung hervorginge. Es kommt also beim Vorliegen eines konkreten Terrorismusgefahrensachverhalts zu einer Gemengelage. Unabhängig von den Theorien des rechtswissenschaftlichen Schrifttums: Welcher Schiene soll das BKA in praxi folgen? Geht Abwehr von Gefahren, also die im Gesetzesentwurf angeführte Straftatenverhütung, hier Strafverfolgung wirklich vor? Belegen nicht schon die neuen heimlichen Ermittlungsbefugnisse eine vor das „\$129a - Verfahren“verlagerte Aufgabe des BKA, Organisationsstrafverfahren zu initiieren? Aus taktischen Gründen soll nach einer Phase polizeirechtlicher „Verdachtsermittlungen“ strafprozessuales Handeln zur effektiveren Abwehr der Gefahr den Vorzug erhalten: Die Rede ist von maximaler Ausnutzung strafprozessualer Befugnisse als Folge polizeirechtlicher Ermittlungen! Nur mit ihnen lassen sich Personenzusammenhänge komplett aufzuklären, Mittäter möglichst rasch festzunehmen, Anschläge verhindern. Nur die Strafprozessordnung kennt extrem intensive Befugnisse: Straftatenverhütung ebnet den Weg zur politischen Strafverfolgung! D a s ist die grundlegende Strategie des neuen BKA - Gesetzes: Im Vorfeld der terroristischen Tat begründet als Verhütung von Straftaten zur Gefahrenabwehr umdeklarierte Vorsorge zur zukünftigen Strafverfolgung ein generelles „Recht auf Ermittlungen zur Suche nach politisch motivierten gefährlichen Personenzusammenschlüssen“. Doch rechtfertigen Tatsachen die Annahme, dass mehr als eine Person eine erhebliche Gefahr für ein hohes Schutzgut der Öffentlichen Sicherheit verursachen könnte, die ein Verbrechen verkörpert, liegen neben der Schutzgutgefährdung mindestens die Voraussetzungen des Verdachts einer Verabredung zum Verbrechen vor. Darüber hinaus ist beispielsweise denkbar: Treffen sich etwa drei als sog. „Gefährder“ eingestufte Zielpersonen und sagt eine Vertrauensperson (VP) der Polizei an, man bespreche einen geplanten Anschlag, lässt sich sofort ein strafprozessualer Verdacht begründen: Heimliche Maßnahmen der StPO, aber auch die Rechte des Beschuldigten, eröffnen sich. So zeigt sich in der Anti - Terror Vorfeldarbeit oberflächlich ein Nebeneinander von Gefahrenabwehr und Strafverfolgung. Ungeachtet juristischer Theorienlehren zur Auflösung dieser Gemengelagen (die h.M. will Gefahrenabwehr v o r Strafverfolgung wissen, was bei bestimmten bedrohten hochwertigen Rechtsgütern, etwa das menschliche Leben, einleuchtet) stellt sich die politische Frage nach der sachgemäßen Führungsverantwortung für Präventivmaßnahmen zur Abwehr von Terrorismus: Nach dem Willen des BMI, soll das reformierte BKA sie ohne Kontrolle der Justiz übernehmen. Erscheint das als eine sachgerechte Lösung? 
Sachleitung der Terrorismusabwehr: Der Staatsanwaltschaft, nicht der Polizei!

Das BMI will über den Weg der Novelle des BKAG die kriminalpolizeiliche Informationserhebungen weiter in das Vorfeld der Straftat verlagern. Doch zur Abwehr möglicher Anschlagsszenarios sowie zur Wahrung von Beschuldigtenrechten sollte Vorfeldarbeit vom $\mathrm{S}$ traf prozes s r e c h t dominiert werden. ${ }^{11}$ Sie muss unter der Sachleitung der Staatsanwaltschaft stehen und im gerichtlichen Verfahren nachprüfbar sein. Strafverfolgung zur Abwehr einer Gefahr für Schutzgüter der Öffentlichen Sicherheit, Gefahrenabwehr durch die Staatsanwaltschaft, speziell die Generalbundesanwältin, bedeutet limitiertes präventives Handeln. Nicht jedoch entgrenzte Vorfeldarbeit, wie sie das BKA kraft der neuen Befugnisse leisten soll. Präventiver Schutz von Rechtsgütern durch Staatsanwaltschaften und eigens für jene Zwecke bereit zu haltende Ermittlungsrichter können anders, als ein auf Vorsorgeermittlungen erweitertes Polizeirecht dem fair trial - Prinzip per se genügen. Die Novelle des BKAG sieht jedoch genau das Gegenteil vor: Den von der Staatsanwaltschaft losgelösten Ausbau kriminalpolizeilicher Ermittlungen. Fraglich erscheint, welchen Charakter dieser Ansatz dem BKA verleiht.

\section{Anti - Terror - Vorfeldarbeit: Straftaten verhüten oder für Strafverfolgung vorsorgen?}

Die Ermittlungsbefugnisse gemäß $\ 20$ a - x BKA - GE sollen es dem BKA ermöglichen, gemäß Art. 73 Nr. 9a GG Straftaten zu verhüten. ${ }^{12}$ Dabei will man nicht an den bisher im BKAG verwendeten Begriff der Straftatenverhütung, einem Unterfall der Gefahrenabwehr, anknüpfen. Handelt es sich also bei den angedachten Befugnissen in Verbindung mit ihrer bisher ungeschriebenen materiellen Grundlage, dem bezüglich $\ 129$ a StGB erweiterten Gefährderbegriff, um Straftatenverhütung, einem Unterfall der Gefahrenabwehr? Straftatenverhütung stellt gemäß $\ 25$ BKAG eine Rechtfertigung der Verarbeitung und Nutzung personenbezogener Erkenntnisse durch das BKA dar. ${ }^{13}$ Sie richtet sich darauf aus, es gar nicht erst zu Straftaten kommen zu lassen. ${ }^{14}$ Es handelt sich um die vorbeugende Bekämpfung von Verbrechen, einem Unterfall der Gefahrenabwehr. Gilt diese Einordnung aber auch für den in $\$ 4$ a Abs. 1 Satz 2 BKA - GE verwendeten Begriff der Verhütung? Aufschluss darüber gibt die bisherige Praxis sog. „Gefahrenermittlungen“ zur Früherkennung islamistischer Zellen, die kraft der neuen Befugnisse ja effektiver ablaufen soll: Staatsschutz und Dienste versuchen seit dem 11. September 2001, Strukturen islamistischer Zellen im Vorfeld eines strafprozessualen Anfangsverdachts, also vor einem versuchten Anschlag, möglichst früh zu erkennen. Diese Systematik hat ein kriminaltaktisches Problem zum Hintergrund: Bundesweit sieht sich der Staatsschutz gezwungen, unterhalb der vom strafprozessualen Anfangsverdacht errichteten Schwelle bzw. ohne das Vorliegen konkreter Gefahrenlagen noch straflose und vor allem nicht gefahrspezifische Vorbereitungshandlungen für Anschläge zu erkennen. Dazu sucht man schlichtweg nach islamistischer Organisationskriminalität. ${ }^{15}$ Denn die potentiellen Täter verhalten sich legal: Sog. „,islamistischen Gefährdern“ zeigen kein für die Einleitung von Ermittlungsverfahren relevantes Alltagsverhalten. Darin besteht ihre Gefährlichkeit, aber auch eine Gefährdung ihrer Grund- und Freiheitsrechte gleichermaßen! Als Surrogat für fehlende strafprozessuale Verdachtsmomente ziehen die Behörden seit dem Frühjahr 2002, nachdem man merkte, das potentielle Täter jede (strafrechtliche) Auffälligkeit sorgsam vermeiden, das Landespolizeirecht heran. Auf dessen Grundlage vollziehen spezielle Dienststellen innerhalb der Staatsschutzabtei- lungen der Landeskriminalämter Ermittlungen zur Früherkennung von vermeintlichen terroristischen Zellen. Die materielle Rechtfertigung solcher „Gefahrenermittlungen“ fußt auf sachverhalts - sowie personenbezogenen Prognosen. Jene verkörpern Aussagen über zukünftiges mögliches (!) Verhalten von Zielpersonen: Ihr Gehalt skizziert aus der ex ante Perspektive des Amtswalters zu erwartende Handlungsabläufe. Grundlage sind bereits gesammelte Erkenntnisse. Doch in jenen finden sich zumeist keine Hinweise auf eine konkrete Gefährdung von Rechtsgütern der öffentlichen Ordnung. Zumeist fielen diese Personen niemals zuvor kriminalpolizeilich - oder wenn, dann nur mit Fällen von unspezifischer Bagatellkriminalität - auf. Und genau darin liegt die Crux von Vorfeldarbeit in Staatsschutzsachen verortet: Im Gegensatz zu ihrem strafprozessualen Pendant fußen polizeirechtliche Ermittlungen auf dem Gehalt menschlicher Voraussagen und daraus abgeleiteter Vermutungen. Sie bilden lediglich eine abstrakte Gefahr ab. Die materielle Rechtfertigung von „Gefahrenermittlungen “ setzt jedoch eine konkrete Gefahr voraus. ${ }^{16}$ An dieser aber fehlt es hinsichtlich des Verhaltens islamistischer Zielpersonen generell: Sonst läge ein Anfangsverdacht nach $\$ 129$ a StGB oder einer anderen Straftat vor. Insoweit zielt die Reform des BKAG darauf ab, durch präventive Ermittlungen ins Blaue hinein vorzusorgen. Dazu erhebt und speichert man seine Daten, konserviert sie in Dateien oder zumindest in einer Handakte. Auf diese Weise produziert der Staat jederzeit abrufbare Intelligence. Dieses seit dem 11. September 2001 bundesweit praktizierte Sammeln von Informationen bedeutet jedoch eines nicht: Gefahrenabwehr. Es handelt sich um strafprozessual vom Bundesgesetzgeber zu regelnde Vorsorge zur Verfolgung zukünftiger, noch unbekannter politisch motivierter Straftaten. Angesichts der seit dem 11. September 2001 entwickelten „Gefahrenermittlungen“ gehen die Autoren des zweiten Referentenentwurfes daher mindestens darin fehl, die in dessen $\mathbb{} 4$ a Abs. 1 Satz 2 BKA - GE enthaltene Aufgabenzuweisung als „Verhütung von Straftaten“ zu bezeichnen. Es handelt sich, wie auch der DeutscheAnwaltverein in einem Gutachten feststellt, um kompetenzwidrig im Polizeirecht verortete Verfolgungsvorsorge. ${ }^{17}$ Kein mustergültig gestalteter Rechtsschutz fängt sie auf.

\section{Weiteres Problemfeld: Rechtsschutz und parlamentarische Kontrolle?}

a) Ex ante gegen Präventivermittlungen: Das AG Wiesbaden - Bollwerk im Vorfeld?

Grundidee der neuen präventiven Funktion, welche das BKA in der Sicherheitsarchitektur einnehmen soll, ist der heimliche Vollzug von Strukturermittlungen. Über den Katalog von dem Amt nach dem BKA - GE zur Verfügung stehenden Maßnahmen würde sich in der Tat jeder Staatsanwalt freuen. Doch welche Instanz setzt die in Art. 19 Abs. IV GG verbürgte Justizgewährleistung um? \20 v Abs. II BKA - GA weist auf das Amtsgericht hin, in dessen Sphäre örtlicher Zuständigkeit das Amt seinen Sitz hat. Das Amtsgericht Wiesbaden also soll Anträge des BKA auf präventive Online - Durchsuchungen, Lausch- und Spähangriffe sowie Telekommunikationsüberwachungen prüfen. Ein Einzelrichter des in Staatsschutzsachen bisher unerfahren Gerichtes soll ex ante - Rechtsschutz gegenüber Ermittlungen garantieren, die nach dem Willen der Polizei in ein \129 a - Verfahren münden. Nicht nur empirischer Erkenntnisse über die Dominanz polizeilicher Begründungsmuster in richterlichen Entscheiden betreffend der Zulässigkeit von Telefonüberwachungen ${ }^{18}$, sondern ihrer Nähe zum Staatsschutzverfahren wegen sollte mindestens eine entsprechende Kammer des OLG, wenn nicht 
sogar der Ermittlungsrichter beim Bundesgerichtshof über auf Prognosen basierende Begehren von stark Grundrechte belastender Intensität wachen.

b) Benachrichtigungen Betroffener weisen zu starke Ausnahmetatbestände auf

Neben der richterlichen Kontrolle erweisen sich Ausnahmetatbestände in $\$ 20 \mathrm{w}$ BKA - GE, die Benachrichtigung über vollzogene Maßnahmen, als zu weit gefasst: Die Autoren des Gesetzes haben diese, die Inanspruchnahme von Rechtsschutz erst ermöglichenden Vorschriften aus der Sicht des um Absicherung seiner erlangten Erkenntnisse auf Vorsorge ausgerichteten Amtes verfasst.

c) Parlamentarische Kontrolle fehlt gänzlich - als Kompensationsmechanismus muss sie her!

Letztlich scheint das BMI die nach Art der Dienste angelegte neue Funktion des BKA nicht im geringsten als die Sphäre des Grundrechtsträgers als belastend zu werten, obwohl nur ein Einzelrichter ex ante die Anträge auf Vollzug der Maßnahmen prüft, während sich eine Benachrichtigung über ihren Vollzug nach $\$ 20 \mathrm{w}$ BKA GE nur zu leicht argumentativ aufschieben lässt. Dieser Erkenntnis ruft förmlich nach einem Mechanismus von Demokratie wahrender Wirkung: Der Gesetzgeber kann ihn in Form eines Gremiums kompensatorisch gestalten. Etwa, indem er in Anlehnung an das die Nachrichtendienste kontrollierende Parlamentarische Kontrollgremium (ParlKGr) eine auf die Vorfeldtätigkeit des zukünftigen BKA ausgerichtete Instanz im Bundestag schafft. Man könnte sie - wie im Hamburg Ende der 90er Jahre - als Polizeikommission bezeichnen. Das Gremium muss zuvörderst Vorgänge analysieren, in denen Benachrichtigungen der Betroffenen unterbleiben. Ebenso prüft es Übermittlungen an ausländische Dienste. Das vor allem im Bezug auf die Folgen der übermittelten Information: Führt sie zu Folter? Werden kraft ihres Transfers in einem anderen Land Personen unrechtmäßig inhaftiert oder ohne Rechtsschutz Repressalien ausgesetzt? Welche Wohnungen betrat das Amt, um in informationstechnische Systeme einzudringen oder Lausch- und Spähvorrichtungen $\mathrm{zu}$ installieren? Folgende Kontrollmodelle erscheinen in Bund und Ländern als realisierbar:

Modell I: Ausweitung der Aufgaben des ParlKGr auf Supervision der Vorfeldarbeit des BKA

Ausweitung der Zuständigkeit der parlamentarischen Kontrollkommission für Gefahrenermittlungen der Staatsschutzabteilung des BKA (Bundesebene): Ihrer Sachnähe zu den ND wegen sollte ein reformiertes ParlKGr auch für die Aufsicht über heimliche Präventivermittlungen zuständig sein. Die Ländern bilden entsprechende Kommissionen in ihren „G-10“ Gremien. In diesem polizeilichen Sachgebiet des reformierten ParlKGr unterlägen ebenso Vorfeldermittlungen von OK- und BtM - Dienststellen einer fallbezogenen ${ }^{19}$ Anzeigepflicht gegenüber dem zuständigen Datenschutzbeauftragten.

Modell II: Polizeibeauftragte beobachten Vorfeldarbeit des Bundes sowie der Länder

Auf Bundesebene wie in den Länder zu implementierenden Polizeibeauftragten $^{20}$, die gleichzeitig Beschwerden über polizeiliche Maßnahmen und / oder Zustände innerhalb der Polizeikultur nachgehen, obliegt die Supervision der gesamten Vorfeldarbeit der Polizei. Sie erstatten den Ausschüssen für Inneres halbjährlich -und bei genau definierten Anlässen- Bericht. Sie prüfen auf die polizeilichen Tätigkeitsfelder Staatsschutz-, OK- und BtM bezogene Präventivermittlungen gleichermaßen.
Modell III: Eine Polizeikommission des Bundestages überwacht die Vorfeldarbeit des BKA

Der Bundestag formt aus den Reihen seiner Abgeordneten, die nicht zwingend Mitglied des Innenausschusses sein müssen oder aus eigens eingestellten Fachleuten eine Kommission. Sie unterliegt keinen Weisungen und hat die gleichen Rechte, wie das ParlKGr derzeit.

\section{Fazit:}

Drei Modelle schlägt der Verfasser aus seiner Sicht vor. Daneben sollte das Polizeirecht der Länder sowie das BKAG eine Vorschrift enthalten, wonach sog. „Gefahrenermittlungen“, also die seit dem 11. September 2001 vom kriminalpolizeilichen Staatsschutz praktizierte Vorfeldarbeit zum Erkennen von Terrorzellen, Fall für Fall einem Richtervorbehalt unterliegen. Daneben ist das Handeln des reformierten BKA so sehr nach Art der Dienste vorsorgend auf Vorfeldarbeit ausgerichtet, was weiche Eingriffsschwellen und eine gewisse Diskretion gegenüber den Betroffenen bedingt, dass dieser Bereich staatlichen Handelns im Wege von Kompensation einer parlamentarischen Form von Aufsicht bedarf. Sie muss institutionell ü b e r die punktuelle Aufsicht des Innenausschusses hinausgehen, welcher eine permanente Kontrolle (wie bezüglich der Dienste) nicht zu leisten vermag. Eine kontinuierliche Analyse von „Gefahrenermittlungen “ erscheint nunmehr geboten.

\section{Das neue BKA: Zusammenfassende Thesen zu einem ent- stehenden Hybrid, einer nachrichtendienstlich arbeitenden Bundeskriminalpolizei}

a) Die Novelle führt eine Erweiterung des bisher rechtswidrigen Begriffs des „islamistischen Gefährders“ ins Polizeirecht ein

\20b Abs. 2 Nr. 1 i.V.m. \4a Abs. 1 S. 2 BKA - GE verkörpert eine von ihrer Weite her bisher nicht gekannte Gefährderdefinition. Jene eröffnet einen viel extensiveren Spielraum des Tätigwerdens, als die seit dem Jahr 2002 verwendete Definition es bisher erlaubt hat. Damit entfernt sich der Entwurf weiter von der Abgrenzung polizeilicher Störer von Nicht - Störern, als das je ein bundesdeutsches Polizeigesetz unternommen hat. Diese Erweiterung bewirkt daneben eine ungeschriebene tatbestandliche Expansion des Unterstützerbegriffs aus $\ 129$ a StGB: Bundeskriminalpolizeirecht erweitert einen Tatbestand des politischen Strafrechts.

b) Straftatenverhütung i.S.d. BKA - GE bedeutet Strafverfolgungsvorsorge, jedoch keine störerorientierte Gefahrenabwehr

Das Reformgesetz verstärkt unter dem Mantel der Verhütung von Straftaten die Strategie des Staatsschutzes, durch Erhebung und Speicherung von Daten und Informationen eine möglichst große Wissensbasis anzulegen. Ein „Knowledge Base Developement“, eine stets erweiterte lernfähige Wissensbasis, ist typisches Merkmal des zeitgemäßen nachrichtendienstlichen Kreislaufes zur Produktion von finished Intelligence. In diesem Sinne sollen die angedachten Befugnisse das BKA von einer kriminalpolizeilichen Zentralstelle nach Art der Dienste in eine Staatspolizei umformen. Zusätzliche Befugnisse sollen ihm keinesfalls die effektivere Verhütung von Straftaten ermöglichen: Das BMI strebt für seine Kriminalpolizei einen Ausbau derer Intelligence - Kompetenzen an. Das ist fatal: Sorgt eine Kriminalpolizei in politischen Strafsachen durch Sammlung von Informationen vor, schwindet ihr auf die Abwehr und Erforschung konkreter Gefahren begrenzter polizeilicher Charakter. Eine geheimdienstliche Nachrichtenpolizei mit Exekutivbefugnissen entsteht.

c) $\$ 20 \mathrm{a}-\mathrm{x}$ setzen Strafverfolgungsvorsorge um, nicht Verhütung von Straftaten als Unterfall von Gefahrenabwehr 
Die kriminalstrategischen Ziele der in $\$ 20$ a-x BKA - GE aufgeführten Ermittlungsmaßnahmen dienen der Vorsorge für die zukünftige Strafverfolgung in Staatsschutzsachen. Die Maßnahmen verkörpern nicht, wie etwa die erkennungsdienstliche Behandlung gemäß $\ 81$ b 2. Alt. StPO, vorbeugende Verbrechensbekämpfung oder die Verhütung von Delikten: Schlichte Vorsorge für zukünftige Strukturermittlungen ist ihr Ziel. Und jenes, ohne dass bereits, wie im Fall von $\mathbb{S} 81 \mathrm{~b} 2$. Alt. StPO, Tatsachen für zukünftig zu erwartende Kriminalitätssachverhalte konkret vorlägen. Verfolgungsvorsorge ordnet sich einer starken Ansicht der Literatur ${ }^{21}$ sowie Entscheidungen des Bundesverfassungsgerichtes ${ }^{22}$ zufolge keinesfalls unter Gefahrenabwehrrecht ein, dessen Setzung gemäß Art. 30, 70 I GG auch nach der Förderalismusreform den Ländern obliegt. Bloße informationelle Vorsorge für zukünftige Strafverfolgung, zählt zum Regelungsgegenstand des Strafprozessrechts. Jenes zu setzen, ist dem Prinzip der konkurrierenden Gesetzgebung zufolge gemäß Art. 74 Abs. I Nr. I GG einzig der strafrechtlichen Kompetenz des Bundesgesetzgebers vorbehalten. Man könnte meinen, dieser habe die Wahl, das BKAG, die Strafprozessordnung oder gar beide Bereiche zur Verortung seiner Befugnisse über heimliche Vorfeldmaßnahmen zu wählen. Doch eine Auswahl obliegt ihm keinesfalls: Der Rspr. ${ }^{23}$ des Bundesverfassungsgerichtes zum niedersächsischen Sicherheits- und Ordnungsgesetz zufolge darf der Bund sein Polizeirecht mit strafprozessual - vorsorgend aufgeladenen Befugnissen ebenso wenig ausstatten, wie die Länder das ihrige. Im Phänomenbereich des islamischen Terrorismus einzig auf die Suche nach Verdachtsmomenten für den Einstieg in strafprozessuale Strukturermittlungen, also auf die Initiierung von Strafverfahren mittels auf Polizeirecht gestützter Ermittlungen (!) ausgerichtet, dienen $\$ 20$ a - x BKA - GE nur vordergründig der Abwehr von Gefahren. Mit ihnen verhütet das BKA keine Straftaten: Vielmehr eröffnen sich heimliche Eingriffe, die für Strafverfolgung vorsorgend Verdachtsmomente sammeln. ${ }^{24}$ Dieser Charakter sprengt die Grenzen des Begriffes polizeilicher Gefahrenabwehr. ${ }^{25}$ Das Staatsrecht endet hier!

d) Die endgültige Abkehr vom Trennungsgebot, wie es die alliierten Mütter und Väter des Grundgesetze von 1945 - 1949 festlegten, läuten Union und Sozialdemokraten zusammen ein

Die in $\mathbb{2 0} \mathrm{a}-\mathrm{x}$ BKA - GE aufgeführten Maßnahmen über heimliche Vorfeldermittlungen haben eines gemeinsam: Sie befördern den Wandel einer Zentralstelle der Kriminalpolizei. Das BKA wandelt sich von einer Nachrichtenpolizei in eine geheim ermittelnde Staatspolizei. Dies aber haben die alliierten Mütter und Väter des Grundgesetzes keinesfalls gewollt, als sie 1949 die Genehmigung des Grundgesetzes von einer strikten organisatorischen sowie befugnisrechtlichen Trennung nachrichtendienstlicher Stellen von solchen der Polizei abhängig machten. ${ }^{26}$

e) Rechtsschutz und Kontrolle: Ein Amtsgericht prüft die Vorfeldermittlungen des BKA, Schwellen nachträglicher Benachrichtigungen werden bewusst hoch konzipiert, eine parlamentarische Kontrolle findet nicht statt

Die Zuständigkeit eines Einzelrichters des AG Wiesbaden spottet Tragweite und Intensität der im BKA - GE aufgeführten Präventivbefugnisse: Mindestens eine dreiköpfige Staatsschutzkammer eines OLG oder der in Terrorismussachen zuständige Ermittlungsrichter beim Bundesgerichtshof sollte kraft entsprechender Erfahrung mit Terrorismussachen für eine Genehmigung der einzig auf Strafverfahren iSd \ 129a StGB abzielenden Vorfeldmaßnahmen zuständig zeichnen. Der kriminalstrategische Ansatz, über einen Kanon heimlicher Überwachungseingriffe Intelligence zu produzieren, fordert ein im Bundestag neu zu schaffendes Kontrollgremium: Es muss analog zum Parlamentarischen Kontrollgremium, welches die Arbeit der Nachrichtendienste prüfen soll, die Vorfeldarbeit des BKA beaufsichtigen, um durch ihre Heimlichkeit bedingte Rechtsschutzverluste zu kompensieren.

Fazit: SPD und CDU setzen mit der Umformung der Sicherheitsarchitektur eine Zäsur, welche Macht in einer Bundesoberbehörde des BMI fragwürdig konzentriert

Mit der Umformung eines von den alliierten Müttern und Vätern des Grundgesetzes kraft ihrer Analyse der Vorfeldarbeit der NS - Sicherheitsarchitektur wohlweislich lediglich als Zentralstelle installierten Bundeskriminalamtes rührt die Große Koalition an einer Wertentscheidung. Nicht einmal 20 Jahre nach der Deutschen Einheit, in denen die Tätigkeit des Ministeriums für Staatssicherheit nicht komplett aufgearbeitet, dessen Vorfeldpraxis - menschen- und bürgerrechtswidrige Repression - nicht umfassend von der Forschung abgebildet worden ist, schafft die Bundesregierung eine zentral aufgestellte Kriminalbehörde, welche nach Art der Nachrichtendienste im Vorfeld konkreter Rechtsgutverletzungen mit umfangreichen Maßnahmen ermitteln wird. Und sie darf präventive Sisitierungen tätigen! Eine derart Macht konzentrierende Verquickung - in Gewahrsamnahmen erfolgen ggf. aufgrund vorangegangener nachrichtendienstlicher Vorfeldarbeit des BKA, wobei sich Rechtsschutz gegen jene Maßnahmen des Amtes und dessen parlamentarische Kontrolle minimal bzw. gar nicht ausgestaltet finden - sollte als Lehre zweier untergegangener totalitärer Staatssicherheitsstaaten nie wieder auf deutschem Boden Staat und Verfassung schützen dürfen. Doch das BMI vereint unverbrämt eine ausgeweitete Produktion von Intelligence mit in die Freiheit der Person eingreifenden Befugnissen unter sich: Ein einmaliger Vorgang, der sich durch keine Bedrohungslage rechtfertigen lässt!

\section{Innenpolitischer Ausblick: Neue Kooperationen eröffnen sich Fachpolitikern!}

Angesichts des skizzierten Paradigmenwechsels ist an der Zeit, der antiliberalen Gestaltung des Politikfeldes „Innere Sicherheit“ durch Christ- und Sozialdemokraten in Bund und Ländern eine fachpolitische Ampelkoalition entgegenzusetzen: Während die Bundesregierung allen Grundsätzen des Polizeiförderalismus sowie den Lehren aus der deutschen Polizeigeschichte in Gestalt von Macht zerteilenden Mechanismen des Rechtsstaatsgedankens - hier speziell dem durch den Wandel des BKA durchbrochenen Trennungsgebot - eine Absage erteilt, lehnen das Die Grünen, die Freien Demokraten sowie Die Linke ab. Hier bieten sich auf Ebene des Bundes sowie der Länder informell sowie den Verhältnissen der Macht geschuldete interparteiliche Kooperationen in Bürgerrechtsfragen an. Zumindest demokratische Sozialisten der Linkspartei sollten von den marktliberalen, jedoch bürgerrechtsbewegten Kreisen der FDP Akzeptanz finden. Die (ehemaligen DDR - Bürgerrechtler unter den) Grünen müssen akzeptieren, dass in der Linkspartei auch undogmatische Linke - Demokraten - aufrichtig für die Freiheitlich Demokratische Grundordnung, für die Bewahrung des Verfassungskerns des Grundgesetzes, für mehr Demokratie und den Schutz von Grundfreiheiten eintreten. Grüne und FDP hegen sicherlich ökologische Differenzen: Doch im Bezug auf Minderheiten und Bürgerrechte trifft man sich, nachdem die Grünen die Zeit als Gestalter Innerer Sicherheit unter Otto Schily verwunden haben. Einzig die Große Koalition hat einen für den Polizeiförderalismus und damit den Rechtsstaatsgedanken 
verhängnisvollen Bund geschlossen. Der in seinem Rechtsempfinden sensible Wähler missbilligt es ihr im Wahljahr 2009 hoffentlich.

\section{Der Verfasser arbeitet für das „Forum Demokratischer Sozialis- mus (FDS) “ und ist als MdB - Mitarbeiter einer Vizepräsidentin des Deutschen Bundestages, Frau MdB Petra Pau (Fraktion Die Linke), tätig.}

\section{Fußnoten:}

1 Statt vieler: Humanistische Union e.V., „Das Bundeskriminalamt - von der zentralen Sammelstelle zur Bundesgeheimpolizei“, Internetauftritt (URL: www. humanistische-union .de) sowie Pressemitteilung v. 12.09.2008. Die älteste deutsche Bürgerrechtsorganisation lehnt das gesamte Vorhaben ausnahmslos ab

2 Statt vieler: „Schäuble: Wichtiger Sicherheits - Baustein“, SZ v. 05.06.2008, S. 5; „Schäuble sieht Dich“, FR v. 05.06.2008, S. 6; „Big Brother marschiert“, ND v. 05.06.2008, S. 1. Der Text des zweiten Referentenentwurfs ist unter der URL des Portals heise.de als PDF - Dokument herunterzuladen: http://www.heise.de/newsticker/BKA-Gesetz-Kritik-aus-CDU-SPDund-Justiz--/meldung/106870/from/rss09)

3 Der Gesetzesentwurf findet sich über die Dokumentensuchmaschine des Bundesrates sowie des Bundestages in: BR - Drs. 16/804/08, Bt - Drs. 16/9588 sowie Bt - Drs. 16/10121 (v. 13.08.2008)

4 73. Sitzung des Bundestags- Innenausschusses am 15.09.2008. Gutachten von Sachverständigen sowie unverlangt eingegangene Stellungnahmen finden sich auf der Webseite des Innenausschusses

5 „Wir sind und bleiben bedroht, “ Interview mit Bundesinnenminister Schäuble, FAZ v. 16.09.2007, S. 4

6 Mit einem Überblick: Tolmein, konkret 8/2008, S. 33; Prantl, SZ v. 15.09.2008, S. 15 sowie v. Denkowski, Kriminalistik 7/2008, 410 - 416

7 Bundesinnenminister Dr. Wolfgang Schäuble (CDU), Rede im Bundestag, 20.06.2008

8 Das Manuskript schloss der Verfasser am 15.09.2008 ab. Es stellt seine persönliche Ansicht dar. Es verpflichtet Mandat und Amt von Frau MdB Pau sowie Die Linke keinesfalls. Für Durchsicht dankt er Frau Rechtsanwältin Barbara Bubb (Wasserburg a. Inn) sowie Frau Dipl. iur (Universität Göttingen) Daniela Piehl (Hamburg)
9 „Das Klima ist nervöser“, Interview mit Innenminister Schäuble, Die Zeit v. 19.07.2007, S. 8

10 Zur Novelle höchst kritisch: Roggan / Bergemann, NJW 2007, 876 (881)

11 Der Verfasser arbeitet seit längerem an einer entsprechenden - eng limitierten - strafprozessualen Vorschrift

12 Vgl. nur die Begründung des hier untersuchten zweiten Referentenentwurfes i.d.F. v. 16.04 .2008 , S. 47

$13 \mathrm{Ahlf} / \mathrm{Daub} /$ Lersch/Störzer, BKAG, Taschenkommentar, 2000, $\$ 25$, Rn. 18

14 Ebenda

15 Kritisch zum Wandel der Vorfeldarbeit: v. Denkowski, „Schutz des Staates im asymmetrischen Konflikt", in: Globaler Terrorismus und Europa, Studien zur Inneren Sicherheit Band 11, Peter Nitschke (Hg.), 2008, S. 167

16 Umfassend: Merten, Datenschutz und Datenverarbeitungsprobleme bei den Sicherheitsbehörden, 1982, S. 135

17 Stellungnahme des Deutschen Anwaltvereins zum BKA - GE, Stellungnahme Nr. 49/2008, S. 9

18 Vgl. nur Albrecht/Dorsch/Krüpe, Rechtswirklichkeit und Effizienz der Überwachung der Telekommunikation, 2004, S. 447

19 Anonymisiert: Aktenzeichen, Sachbearbeiter, Anordnender, Dienststelle, Gegenstand der Ermittlung

20 Jene Institution demokratisch - parlamentarischer Kontrolle sollte auf Bundesebene und in den Ländern geschaffen werden

21 Puschke / Singelnstein, NJW 49/05, 3534; (mwN) Kutscha, Martin, „Innere Sicherheit und Freiheitsrechte“, in: Handbuch zum Recht der Inneren Sicherheit, Roggan / Kutscha (Hrsg.), 2. Aufl. 2006, S. 82; v, Denkowski, „La protección estatal en los conflictos asimétricos: ¿Continúa el Derecho policial de los Länder alemanes, tras el 11 de septiembre, con el Derecho penal político antiliberal? “, in: Dereche penal del enemigo. El discurso penal de la exclusion, 2006, Cancio - Melia / Jara - Diez (Hg.), Buenos Aires

22 BVerfG NJW 05, 2605; BVerfGE 103, 21 (30), BVerfGE NJW 01879 (880); Stimmen der a.A.: Bgb. LVerfG LKV 96, 450 (451); BayVerfGH, NVwZ 96, 166; MVVerfGH LKV 00, 149 (151); Kniesel, ZRP 89, 332

23 Vgl. nur: BVerfG, Urteil v. 27.07.2005, Az. 1 BvR 668/04, Absatz-Nr. $96 \mathrm{ff}$. ( $\$ 33$ a SOG Niedersachsen)

24 So auch: Stellungnahme des Deutschen Anwaltvereins zum BKA - GE, Stellungnahme Nr. 49/2008, S. 9

25 Die Gestaltung ihrer strafprozessualen Variante bliebe dem Bundesgesetzgeber dagegen gestattet, verwürfe das Bundesverfassungsgericht die Maßnahme nicht kraft der Problematik absolut geschützter Kernbereiche

26 Grundlegend: v. Denkowski, „Polizei Verfassungsschutz Trennungsgebot“, Kriminalistik 3/2008, $176 \mathrm{ff}$

\section{Strafrechtliche Verfolgung von Subventionsbetrügereien in der EG \\ Ingo E. Fromm und Jens Naussed}

\section{Einleitung}

\subsection{Einführung in die Problematik}

Kriminelle Bestrebungen Einzelner und ganzer Banden, die sich durch betrügerische Aktivitäten finanzielle Vorteile aus dem Gemeinschaftsvermögen beschaffen wollen, gab es schon vor mehr als 50 Jahren. Die allerersten Gedanken an die Schaffung der elementaren Voraussetzung der strafrechtlichen Ahndung von Verstößen gegen Vorschriften des Gemeinsamen Marktes stammen aus dem Jahre 1962. ${ }^{1}$ Aktuell sieht der EU-Haushalt 2008 Gesamtausgaben von ca. 120,3 Mrd. € vor und stellt somit einen sehr verlockenden Anreiz für kriminelle Machenschaften dar. ${ }^{2}$ Proportional zum wachsenden Gemeinschaftsbudget haben die Mitgliedsstaaten immer mehr Betrugsfälle gemeldet. ${ }^{3}$ Der von der Kommission errechnete Gesamtschaden ist mit $1,74 \mathrm{Mrd} . €^{4}$ mittlerweile derart exorbitant hoch, dass er allein den Gesamthaushalt einzelner Mitgliedstaaten übersteigt. Wie hoch der Schaden wegen Subventionsbetrugs in der EG insgesamt ist, ist unklar. Einige Schätzungen gehen davon aus, dass bis zu $10 \%$ des Gesamthaushaltes durch Subventionsbetrug verlo- ren gehen. ${ }^{5}$ Neben einigen Autoren hat auch der EU-Rechnungshof darauf hingewiesen, dass die Kommission ihre Kontrollanstrengungen verstärken müsste. ${ }^{6}$ Zur effektiven Abschreckung könnte auch die Einführung von europaweit verbindlichen strafrechtlichen Vorschriften diese Situation erheblich verbessern. Die Tatbestände der jeweiligen Mitgliedsstaaten sind zurzeit inhaltlich noch sehr unterschiedlich ausgeführt. ${ }^{7}$ In Deutschland ist beispielsweise bereits leichtfertiges Handeln der Subventionsbetrüger mit Strafe bedroht ( $\int 264$ IV StGB). Im Vergleich dazu kennt das spanische Strafrecht lediglich einen betrugsähnlichen Tatbestand, der nur vorsätzliche Schädigungen unter Strafe stellt. ${ }^{8}$

Transnationale organisierte Betrüger können sich so die unterschiedlichen Rechtssysteme zunutze machen, zumal zurzeit mangels einer eigenen originären Strafrechtskompetenz der EG nur eine Bestrafung durch die einzelnen Mitgliedstaaten erfolgen kann. Deshalb fordern einige Autoren eine zumindest ähnliche Strafbarkeit und Strafverfolgung innerhalb der Mitgliedsstaaten. ${ }^{9}$ Problematisch ist in diesem Zusammenhang aber, dass viele Mitgliedsstaaten das Strafrecht 